\title{
eRNAs and Superenhancer lncRNAs Are Functional in Human Prostate Cancer
}

\author{
Xiaona Zhang, ${ }^{1,2,3}$ Panpan Pang, ${ }^{2,3,4}$ Min Jiang, ${ }^{1,2,3}$ Qunfa Cao, ${ }^{2,3}$ Huili Li, $^{2,3}$ Yi Xu, \\ Yao Li $\mathbb{D}^{4}{ }^{4}$ Xue Chen $\mathbb{D}^{1},{ }^{1}$ and Junsong Han $\mathbb{D}^{2,3}$ \\ ${ }^{1}$ Department of Pathology, Tongji Hospital, Tongji University School of Medicine, Shanghai, China \\ ${ }^{2}$ National Engineering Center for Biochip at Shanghai, Shanghai, China \\ ${ }^{3}$ Shanghai Biochip Corporation, Shanghai, China \\ ${ }^{4}$ Shanghai Engineering Research Center of Industrial Microorganisms, School of Life Science, Fudan University, Shanghai, China \\ ${ }^{5}$ Yueyang Hospital of Integrated Traditional Chinese and Western Medicine, Shanghai University of Chinese Medicine, \\ Shanghai, China
}

Correspondence should be addressed to Yao Li; yaoli@fudan.edu.cn, Xue Chen; chenxue20200101@163.com, and Junsong Han; hanjsong@21cn.com

Received 23 June 2020; Revised 27 July 2020; Accepted 14 August 2020; Published 24 September 2020

Academic Editor: Mingjun Shi

Copyright ( $) 2020$ Xiaona Zhang et al. This is an open access article distributed under the Creative Commons Attribution License, which permits unrestricted use, distribution, and reproduction in any medium, provided the original work is properly cited.

\begin{abstract}
Prostate cancer ( $\mathrm{PCa}$ ) is one of the most commonly diagnosed cancers in males worldwide. lncRNAs (long noncoding RNAs) play a significant role in the occurrence and development of PCa. eRNAs (enhancer RNAs) and SE-lncRNAs (superenhancer lncRNAs) are important elements of lncRNAs, but the role of eRNAs and SE-lncRNAs in PCa remains largely unclear. In this work, we identified 681 eRNAs and 292 SE-lncRNAs that were expressed differentially in PCa using a microarray. We also found that eRNAs transcribed from active open chromatin had significantly higher expression than those from active closed chromatin, and SE-lncRNAs had a little higher expression than eRNAs. Next, we constructed a transcriptional regulation network that eRNA-related enhancer and the target genes shared the same TF-binding motifs. Further, we investigated whether CTCF played a role in mediating the transcriptional regulation network. eRNAs, especially those that regulate androgen response genes, may be candidates for prognostic biomarkers and therapy targets. Our work provides a new perspective for developing medical treatments and therapies for prostate cancer.
\end{abstract}

\section{Introduction}

In the last decade, it has been shown repeatedly that the genomes of many species are transcribed pervasively to produce noncoding transcripts [1]. A class of noncoding RNAs, typically more than 200 nucleotides, is referred to as long noncoding RNA (lncRNA). IncRNAs have been proposed to carry out diverse functions that include transcriptional regulation at multiple levels, organization of nuclear domains, and regulation of proteins or RNA molecules [25]. According to the characteristics of IncRNA transcripts, there are several types of lncRNAs, which include intergenic transcripts, sense or antisense transcripts that overlap other coding genes, and eRNAs [6]. A novel class of enhancer transcribed lncRNAs, referred to as enhancer RNA (eRNA) [7-11].

Enhancers [12] are key cis-acting gene regulatory elements in eukaryotes, which can effectively promote the expression of target genes [13]. They can sustain precise control of transcription by serving as binding platforms for transcription factors (TFs) and cofactors that regulate productive transcription at core promoters during development; their misregulation contributes to human diseases [14-16]. Enhancers are generally found within accessible chromatin [14-16]. Nevertheless, they can either be located close to the core promoter or distal to their target genes [17-19]. Genome-wide analysis suggests that enhancers may be transcribed generally $[7,20]$. eRNAs are expressed in a tissue- 
specific manner $[21,22]$, and they play an important role in enhancer activity and regulating gene transcription by diverse mechanisms [23-28]. eRNAs are involved in the enhancement of transcription. For instance, eRNA production from p53-bound enhancer regions (p53BERs) was required for efficient enhancement of p53 transcription [24]. Nuclear receptors, such as estrogen receptor (ER), androgen receptor (AR), and Rev-erbs-related eRNAs, have been demonstrated that eRNA knockdown can lead to correlate changes in target gene expression [23, 25, 29-31].

Superenhancers (SE) consist of large clusters of transcriptional enhancers that are distinct from the typical enhancers in their ability to activate cell-type and tissue-specific genes and result in a higher susceptibility for disease. SE-lncRNA CCAT1-L interacted with CTCF and modulated chromatin conformation at the MYC loop regions in colorectal cancer [32]. SE-lncRNA UCA1 enhanced the AMOT-YAP interaction to promote YAP dephosphorylation and nuclear translocation to activate YAP target genes in epithelial ovarian cancer [33].

Prostate cancer $(\mathrm{PCa})$ is one of the most commonly diagnosed cancers in males worldwide $[34,35]$, and it remains the third most common killer in men with cancer [36]. The role of eRNAs and SE-lncRNAs in prostate cancer remains largely unclear. In this study, our custom-designed noncoding RNA microarrays were used to compare the expression profiling of prostate tumors and corresponding adjacent normal prostate tissues to identify the differentially expressed eRNAs and SElncRNAs and to explore their regulated functions.

\section{Materials and Methods}

2.1. Sample Preparation. Tumor and corresponding adjacent normal prostate tissues were obtained from three $\mathrm{PCa}$ patients at the Fudan University Shanghai Cancer Center. Those patients did not receive any preoperation treatment. The study was approved by the Research Ethics Committee of Fudan University Shanghai Cancer Center. Informed consent was provided by all patients. All samples were collected and used for gene expression analysis by microarray and qRT-PCR. Total RNA was isolated from tumor and corresponding adjacent normal prostate tissues using TRIzol Reagent (Life Technologies, Carlsbad, CA, US), according to the manufacturer's instructions. All samples were checked for a RIN number to inspect RNA integration by an Agilent Bioanalyzer 4200 (Agilent Technologies, Santa Clara, CA, US).

2.2. Microarray Design. A custom microarray was designed by eArray, a web-based application for Agilent's custom microarray design from Agilent Company. The microarray contains $93221 \operatorname{lncRNAs}$ and 27482 mRNAs; the probe length is $60 \mathrm{nt}$. The entire set of protein-coding mRNA from Refseq of NCBI and lncRNA contents are based on the database of NCBI, UCSC, Ensembl, GENCODE, LNCipedia, lncRNAdb, and NONCODE.

2.3. Microarray Assay and Data Analysis. Qualified total RNA was further purified by a RNeasy microkit
(Cat\#74004, QIAGEN, GmBH, Germany) and a RNaseFree DNase Set (QIAGEN, GmBH, Germany). Purified RNA was then used to generate fluorescence-labeled cRNA for the SBC human ceRNA array $(4 \times 180 \mathrm{~K})$. The hybridization solution was prepared according to the in situ hybridization kit plus (Agilent Technologies, Santa Clara, CA, US). Hybridization was carried out using a custom microarray at $60^{\circ} \mathrm{C}$ for $18 \mathrm{~h}$. After hybridization, the slide was scanned on an Agilent Microarray Scanner (Agilent Technologies, Santa Clara, CA, US). Data were extracted with Feature Extraction software 12.1 (Agilent Technologies, Santa Clara, CA, US). The raw data were normalized by the limma package in $\mathrm{R}$ software, and the quantile algorithm was used. The normalized signal was calculated by $\log 2$.

Ratios were calculated between the two groups using Student's $t$-test and fold change. Hierarchical clustering was applied to elucidate the diacritical eRNA expression pattern. Statistically significant eRNAs that were expressed differentially were displayed using a volcano plot that was filtered by fold change $\geq 2, p$ value $<0.05$.

2.4. Identification of $e R N A$ and SE- $\ln c R N A$. Enhancers were downloaded from three sources, and redundancy was removed: ENCODE DHS (DNase I hypersensitivity sites) data were downloaded from the DENdb database (https:// www.cbrc.kaust.edu.sa/dendb/) [37], and H3K27ac CHIPseq and DNase-seq data were downloaded from ENCODE (https://www.encodeproject.org/) [38]. Then, active enhancers were identified following the rule: active enhancer must be open chromatin and have high H3K27ac max-Zs. If they were TSS proximal, they also had to have low $\mathrm{H} 3 \mathrm{~K} 4 \mathrm{me} 3$ max-Zs. Furthermore, each enhancer was classified as being proximal $(\leq 2 \mathrm{~kb})$ or distal $(>2 \mathrm{~kb})$ to the nearest GENCODE annotated TSS. The third part of active enhancers that was identified by STARR-seq in LNCaP cells was downloaded from GSE82204 (https://www.ncbi.nlm.nih.gov) [14]. STARR-seq is a massively parallel reporter assay to identify active enhancers based directly on their activity in entire genomes, so the enhancers identified by STARR-seq may be in closed chromatin. Superenhancers were downloaded from superdb (https://sourceforge.net/projects/superdb/). eRNAs and SE-lncRNAs were identified from lncRNAs using a SBC human ceRNA array where a minimum of one nucleotide overlapped with above enhancers and superenhancers using BEDTools [39].

2.5. Prediction of eRNA Target Genes. Differentially expressed eRNAs were selected for target gene prediction as described previously. The Refseq genes within a $300 \mathrm{~kb}$ window upstream or downstream of eRNAs were considered to be target genes using ClosestBed functionality in BEDTools. Corresponding target genes that were expressed differentially in the microarray with eRNA were selected further.

2.6. Analysis of Functional Enrichment of Target Genes. To better understand the biological functions and pathways of the differentially expressed target genes, Gene Ontology (GO) was analyzed using the R/Bioconductor package clusterProfiler, and the Kyoto Encyclopedia of Genes and 
Genomes (KEGG) pathway was analyzed by DAVID (https://david.ncifcrf.gov/). Functional enrichment analysis was based on the threshold of $p$ value $<0.05$.

2.7. TCGA Data Analysis. To validate eRNA microarray results, RNA-seq data that were generated with 33 paired tumors from patients with nonrecurrent prostate cancer and patients with normal tissues were obtained from TCGA (https://tcga-data.nci.nih.gov/tcga/), which was imputed on an Illumina HiSeq RNA-seq platform. Statistical analysis was done using the Mann-Whitney-Wilcoxon test with RPKM for all tumors and normal tissues. The differentially expressed RNAs of the data set were filtered by fold change $\geq 2, p$ value $<0.05$. The differentially expressed eRNAs were identified based on the Integrated Annotation of Ensembl (http://asia.ensembl.org/index.html) and microarray eRNAs. RNA-seq data and clinical information of PCa are publicly available and in open access platforms. Therefore, approval by the local ethics committee was not needed.

2.8. $q R T-P C R$ Assay. The RNA of each group was reverse transcribed to cDNA with the PrimeScript RT reagent Kit (TaKaRa, Dalian, China). Quantitative real-time PCR assay was performed with SYBR Premix Ex Taq ${ }^{\mathrm{TM}}$ II (TaKaRa, Dalian, China) and a 7900 system (Applied Biosystems, Carlsbad, CA, U.S.A.) in triplicate for each sample, according to the manufacturer's instructions. All the primer sequences are listed in Supplementary Table 1 the raw data are listed in Supplementary Table 3. The expressions of specific genes were calculated using the comparative $2^{-\Delta \Delta \mathrm{Ct}}$ method with $\beta$-actin as a calibrator.

2.9. Prediction of Transcription Factors (TF) and Construction of Interaction Networks. The prediction of potential TFs aimed at target genes and enhancers was done by HOMER (http://homer.ucsd.edu/homer/) motif analysis for the promoter of eRNA target genes and eRNA-related enhancers. For target genes, the search areas included DNA $2 \mathrm{~kb}$ upstream of each gene locus, and the search area for enhancers was its chromatic locus. The interaction of enhancer (eRNA) and target gene promoters that shared the same TF-binding motifs was confirmed by chromatin interaction region data that were generated with $3 \mathrm{C}, 4 \mathrm{C}$, and 5C and a ChIA-PET assay from DENdb database [37]. The selected enhancer- (eRNA-) TF target gene coordination formulated the interaction networks using Cytoscape 3.4.0 software (Agilent and IBS, Santa Clara, CA, U.S.A.). The degree represented the importance of eRNAs, enhancers, TFs, and target genes in the network.

2.10. CTCF ChIP-seq Data Analysis. To determine whether CTCF contributed to the mediation of chromatin looping of enhancer-target gene promoters, CTCF ChIP-seq data in LNCaP cells were downloaded from ENCODE. We then measured whether CTCF enrichment in both enhancers and promoters of 147 eRNA- (enhancer-) TF target genes was coordinated as previously analyzed by BEDTools.

2.11. Public Data Download. ENCODE DHS and chromatin interaction region data that were generated with $3 \mathrm{C}, 4 \mathrm{C}$, and
5C and a ChIA-PET assay were downloaded from the DENdb database (https://www.cbrc.kaust.edu.sa/dendb/). H3K27ac CHIP-seq in the PC-3 cells, CTCF ChIP-seq in LNCaP cells, and DNase-seq data were downloaded from ENCODE (https://www.encodeproject.org/). STARR-seq in LNCaP cells was downloaded from GSE82204 (https://www .ncbi.nlm.nih.gov). Superenhancers were downloaded from superdb (https://sourceforge.net/projects/superdb/). The transcriptome data and corresponding clinical information of 33 nonrecurrent prostate cancer $\mathrm{PCa}$ patients were obtained from TCGA data (https://tcga-data.nci.nih.gov/ tcga/), which was imputed on the Illumina HiSeq RNA-seq platform.

\section{Results}

3.1. Identification of Differentially Expressed eRNAs in Prostate Cancer. We designed a custom microarray containing $93221 \mathrm{lncRNAs}$ and 27482 mRNAs using eArray, a webbased application for Agilent's custom microarray design, namely, SBC human ceRNA array. To identify eRNAs and SE-lncRNAs contained in our custom-designed noncoding RNA microarray, we downloaded enhancers from ENCODE, the DENdb database, and STARR-seq data from the GEO database (GSE82204). DENdb-integrated enhancers were predicted by five different methods (CSI-ANN, Segway, ChromHMM, RFECS, and the ENCODE integrative annotation) that generated an enriched catalogue of putative enhancers for each of the analyzed cell lines [37]. A total of 470,432 ENCODE DHS that supported enhancers were obtained from the DENdb database. The ENCODE (Encyclopedia of DNA Elements) project identified and analyzed all regulatory elements in the human genome [38]. The high-quality histone mark enrichment ChIP-seq and DNase-seq data were downloaded. A total of 55,603 active enhancers were identified from ENCODE data in PC-3 cells. Whole-genome STARR-seq is a method that can obtain active enhancers more reliably. Liu et al. identified 94,527 active enhancers by whole-genome STARR-seq in LNCaP cells [14].

All identified enhancers that overlapped lncRNAs contained in the microarray were further identified as eRNAs [40]. Next, we integrated the three parts of eRNAs after removing redundancy, and 43,680 eRNAs were identified (Figure 1(a); Table 1).

To investigate transcriptional networks of all regulatory elements and to uncover the role of eRNAs and SElncRNAs in prostate cancer, we assessed the eRNA and mRNA expression patterns in prostate cancer. Three paired prostate tumors and corresponding adjacent normal tissues were analyzed using the microarray.

For quality control, we compared the expression of all eRNAs and mRNAs obtained from all of the samples by PCA to ensure that variance in the expressions between prostate tumors and corresponding adjacent normal tissues existed, and the conditions were similar (Figure 1(b)). Then, 681 differentially expressed eRNAs and 1220 differentially expressed mRNAs were identified by Student's $t$-test using the threshold of $p$ value $<0.05$ 

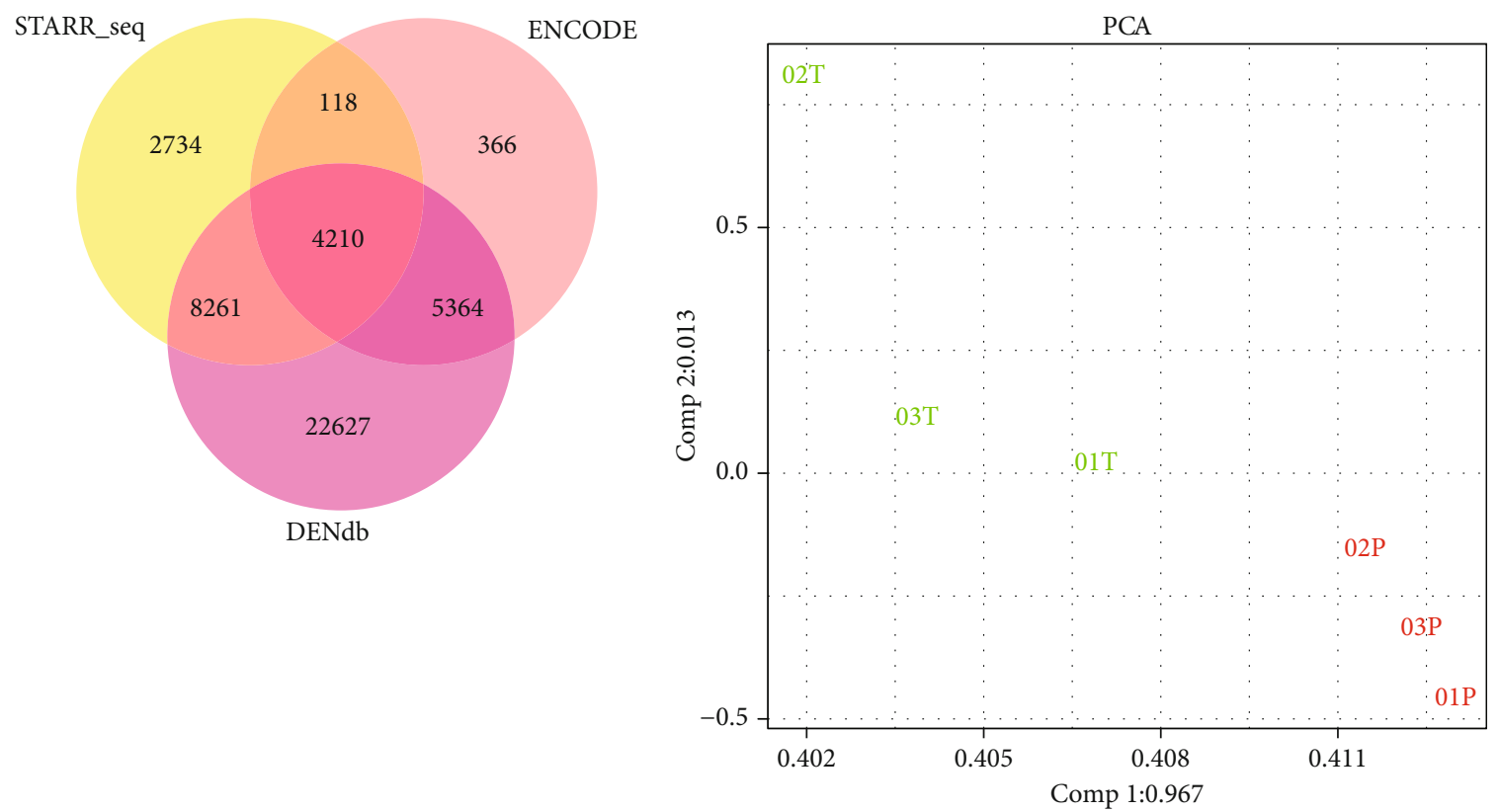

(a)

(b)

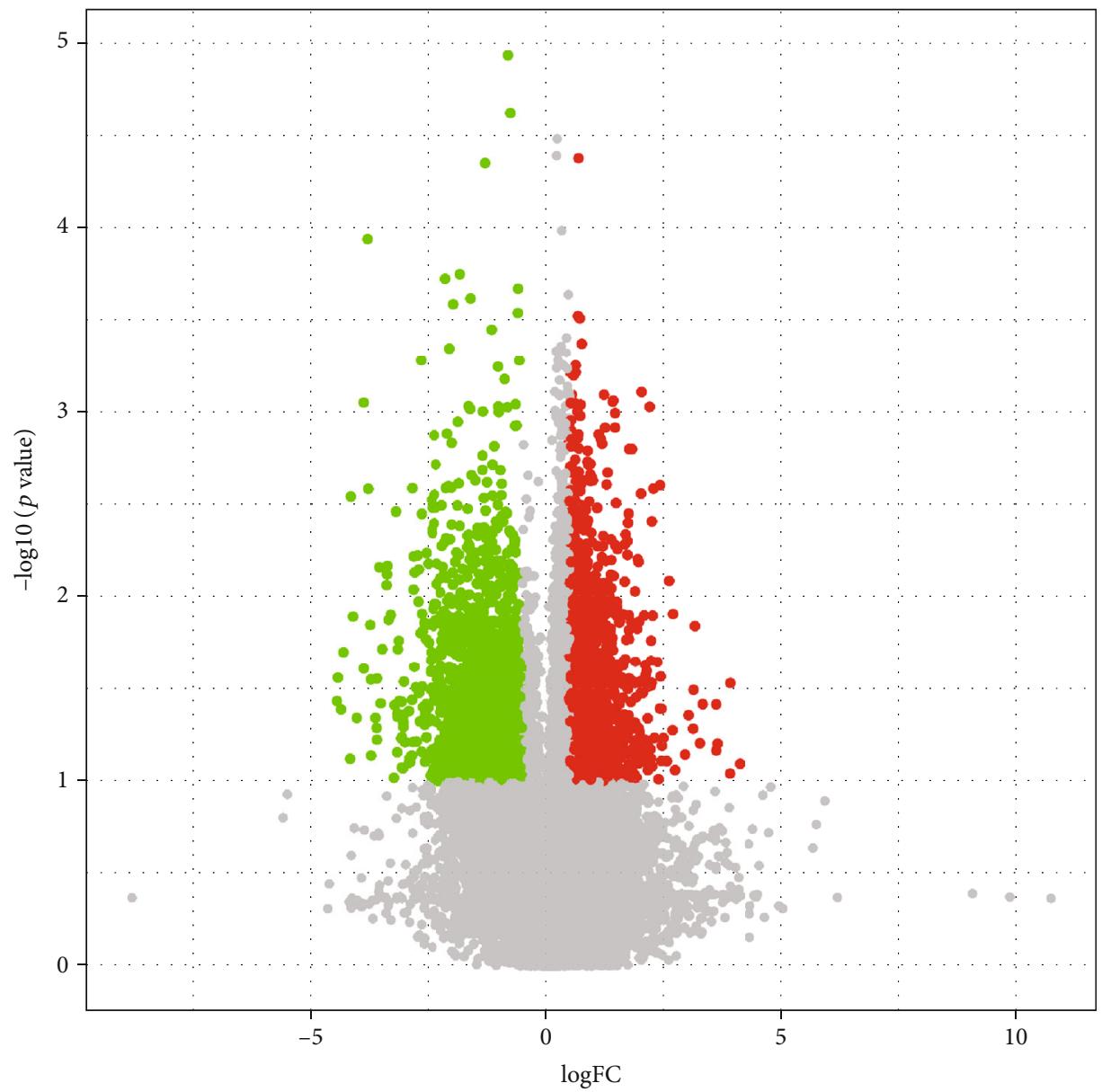

(c)

FIgURe 1: Continued. 


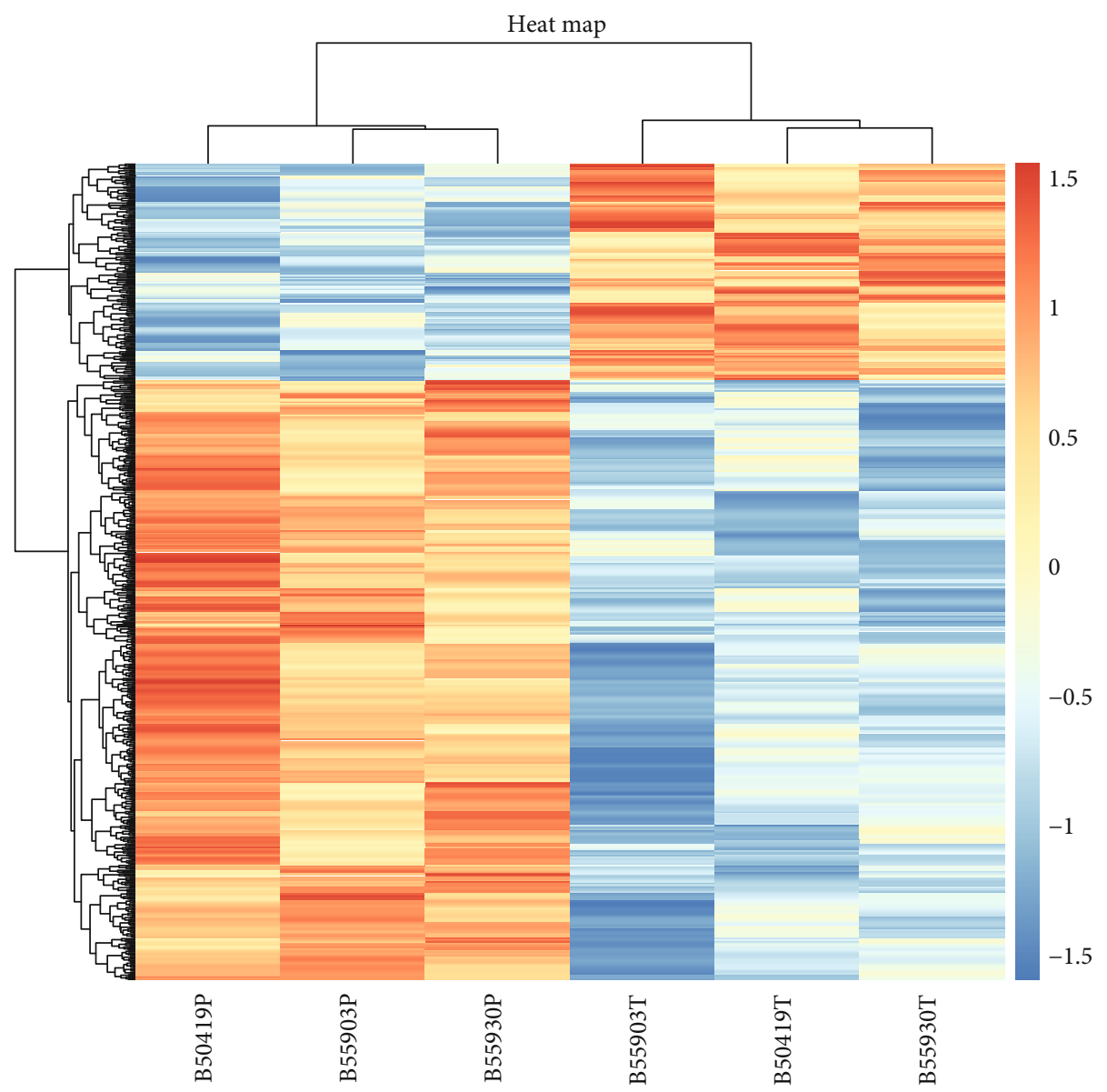

(d)

FIGURE 1: Overview of differentially expressed eRNAs in prostate cancer. (a) Venn diagram showing the overlap of eRNAs identified from DENdb, ENCODE, and STARR-seq (GSE82204). (b) Principal component analysis (PCA) score plots, the expression pattern of each sample from the same condition were similar, which showed the high reliability and reproducibility of the microarray data. (c) Differentially expressed eRNAs in a microarray were filtered by a volcano plot that was filtered by fold change $\geq 2, p$ value $<0.05$, where red shows upregulated and green shows downregulated. (d) A heat map of differentially expressed eRNAs in a microarray, where red shows upregulated and blue shows downregulated.

TABLE 1: The sources of eRNAs and differentially expressed eRNAs in a microarray.

\begin{tabular}{lcccc}
\hline & Total & DEN database & ENCODE & GSE82204 \\
\hline All eRNA sources & 43680 & 40462 & 484 & 2734 \\
Differentially expressed eRNA sources & 681 & 658 & 3 & 20 \\
\hline
\end{tabular}

and fold change $\geq 2$ (Figures 1 (c) and 1(d); Table 1). The distributions of differentially expressed eRNAs were analyzed based on three criteria: classification, length, and chromosome locus (Figures 2(d)-2(f)). The GEO accession number for the microarray data is GSE155056.

3.2. The Expression of eRNAs Transcribed from Active Enhancers. eRNAs that were transcribed from active enhancers were thought to be active eRNAs. We analyzed the expression of active eRNAs in our microarray data, the related active enhancers identified in the LNCaP cells base in STARR-seq data (GSE82204) and identified in the PC-3 cells base in ENCODE data. There was no significant difference in the average signal between active eRNAs identified in ENCODE (average signal: 5.45) and all eRNAs (average signal: 5.54), but the average signal of active eRNAs identified in STARR-seq was 5.72, which was a little higher than all eRNAs.

STARR-seq is a massively parallel reporter assay to identify active enhancers based directly on their activity in entire genomes; the enhancers identified by STARRseq may be in open or closed chromatin. As the work of Liu et al. showed, associated analysis of STARR-seq and DNaseI-seq data (ENCODE) from LNCaP cells found that only $11,980(12.7 \%)$ of the 94,527 active 


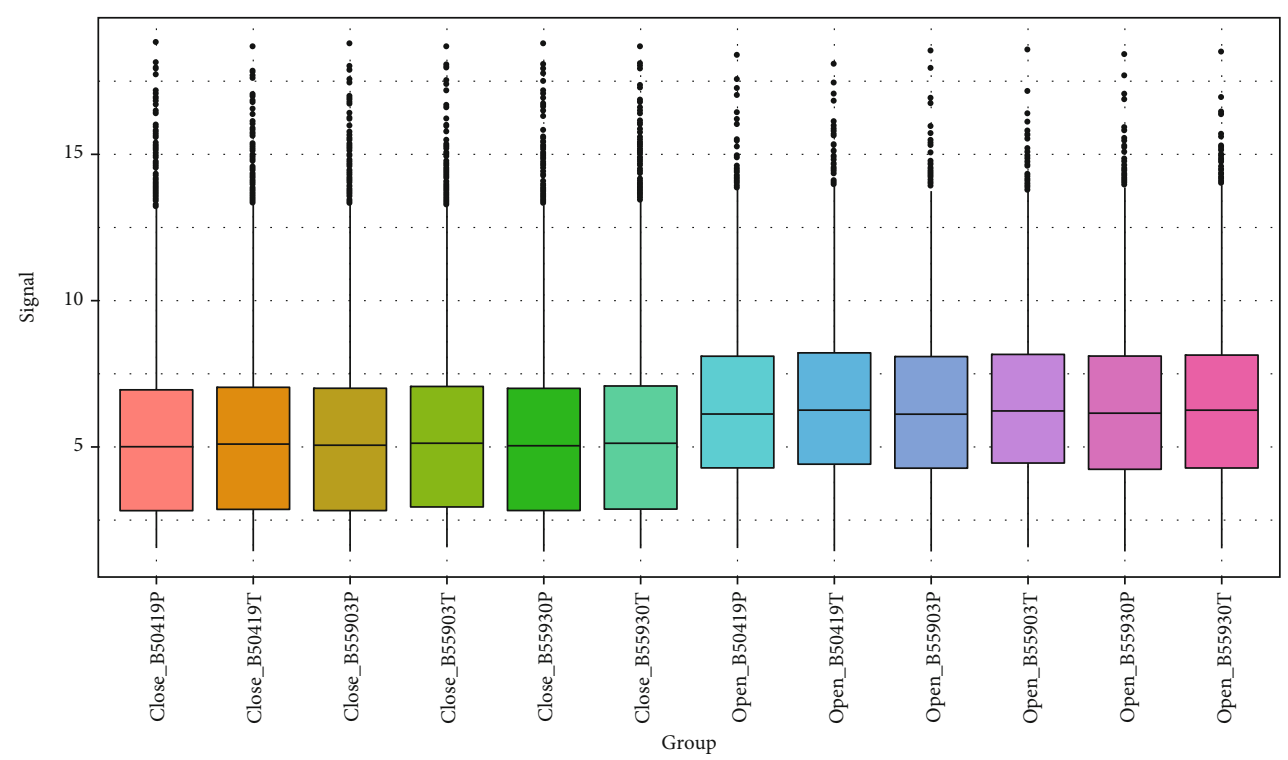

Group
追 Close_B50419P
보 Open_B50419P
官 Close_B50419T
追 Open_B50419T
官 Close_B55903P
官 Open_B55903P
官 Close_B55903T
追 Open_B55903T
官 Close_B55930P
巨Open_B55930P
Ð Close_B55930T
부 Open_B55930T

(a)

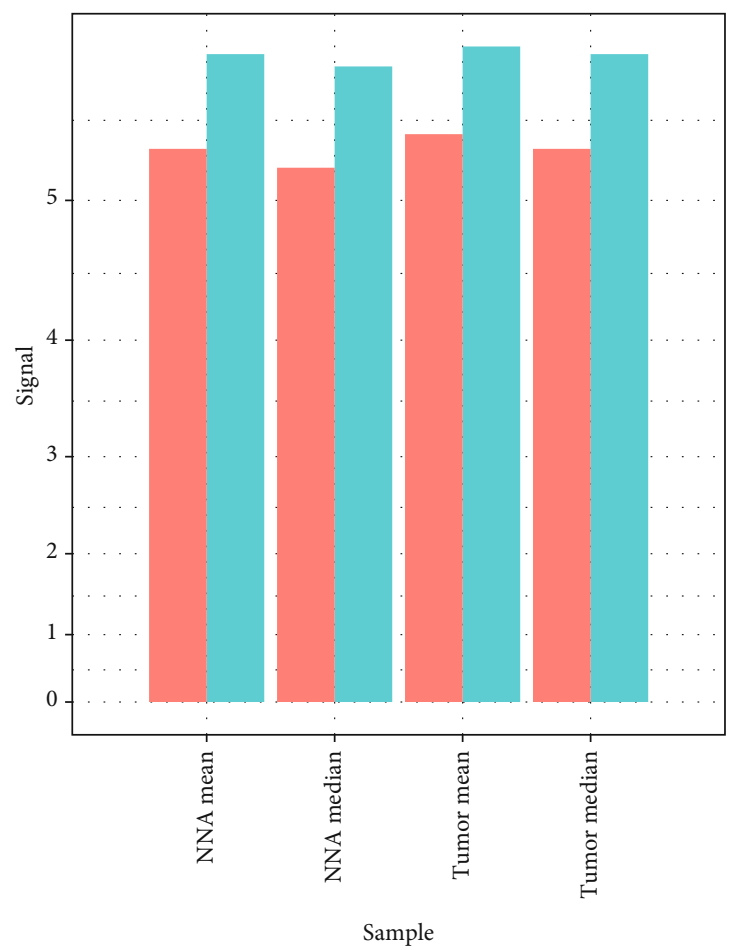

Group

eRNA only

SE-lncRNA

(b)

Figure 2: Continued. 


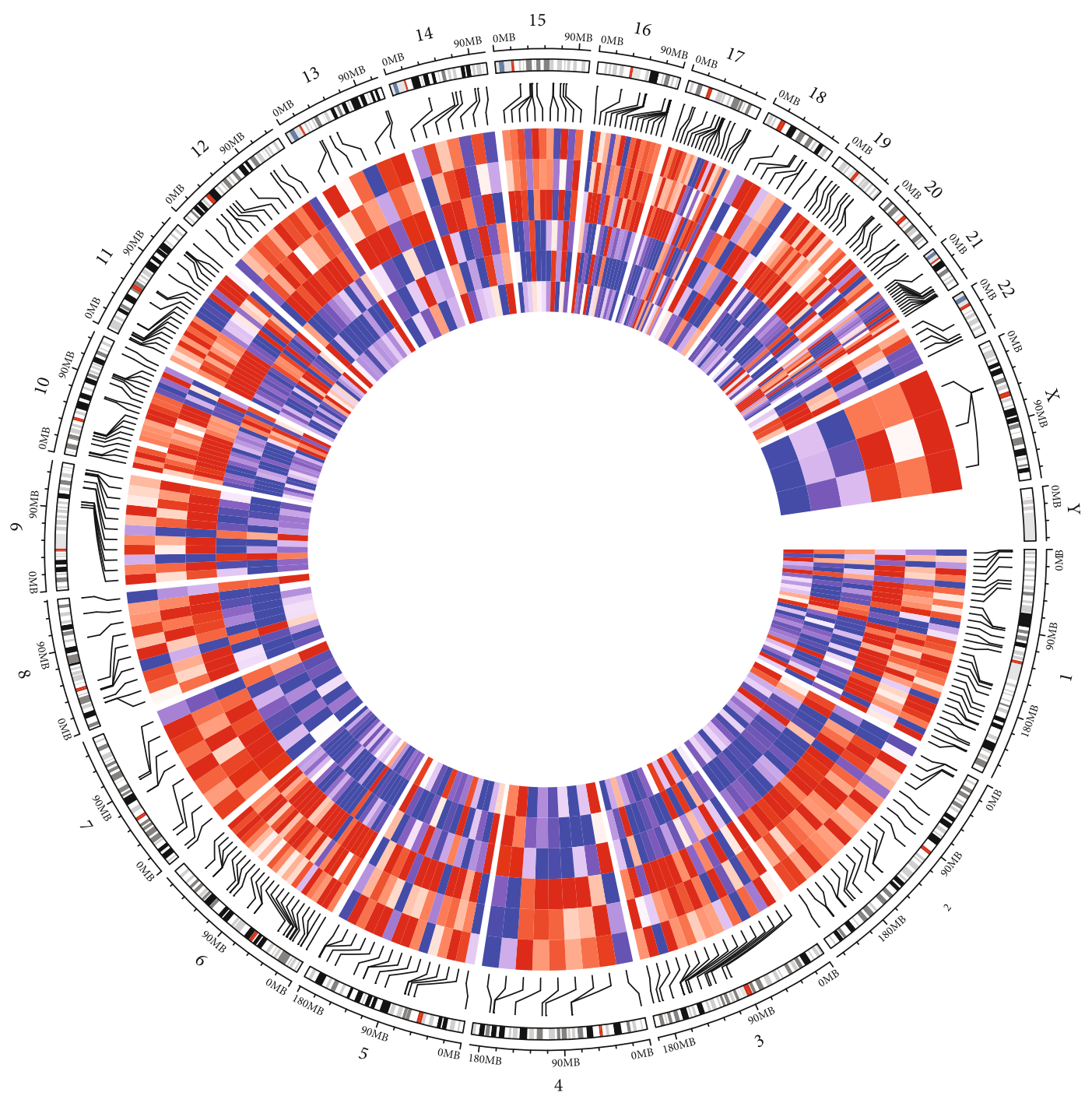

(c)

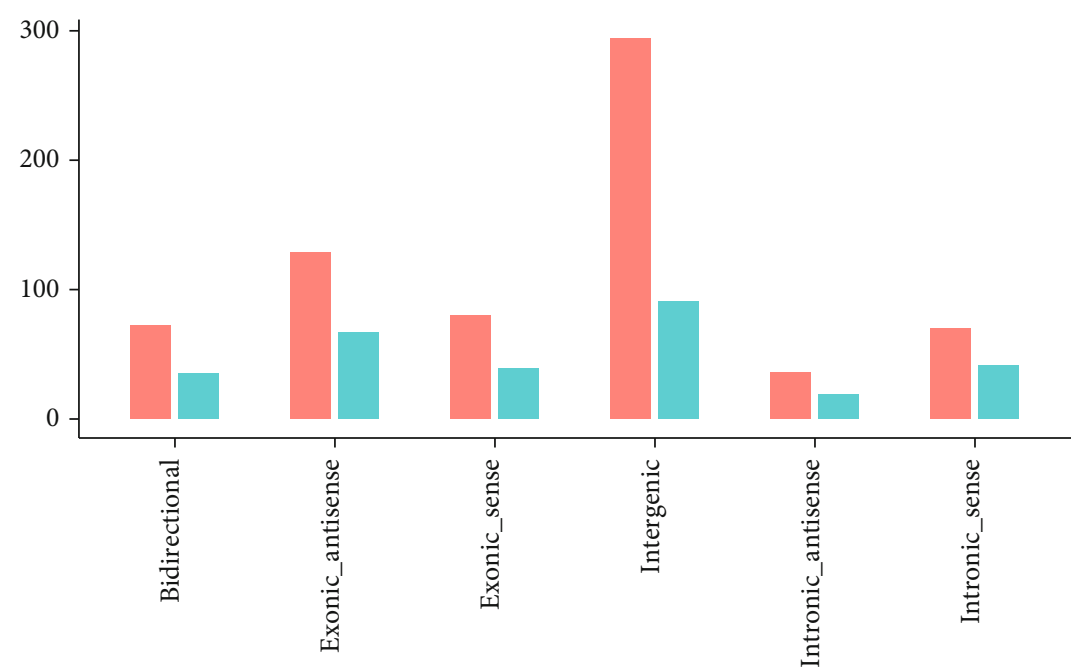

eRNA

SE-lncRNA

(d)

Figure 2: Continued. 
SE_lncRNA_len_distri (total length: 292)

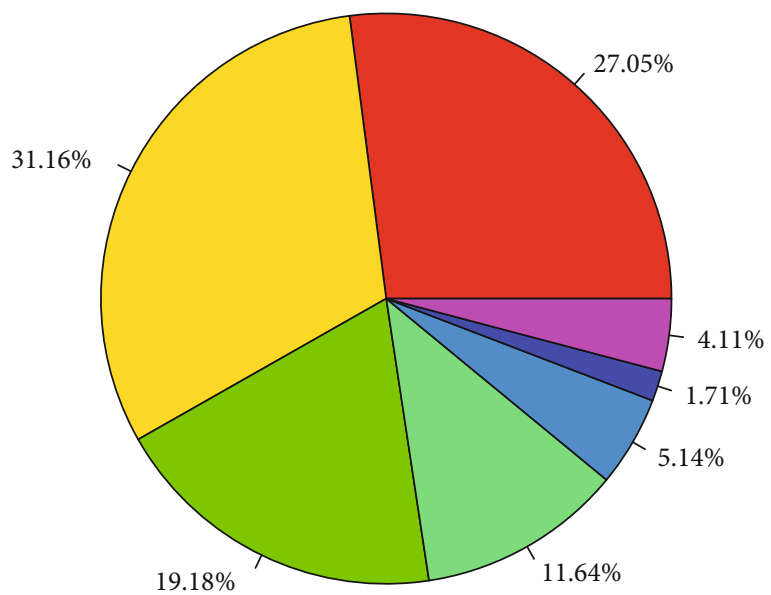

$19.18 \%$

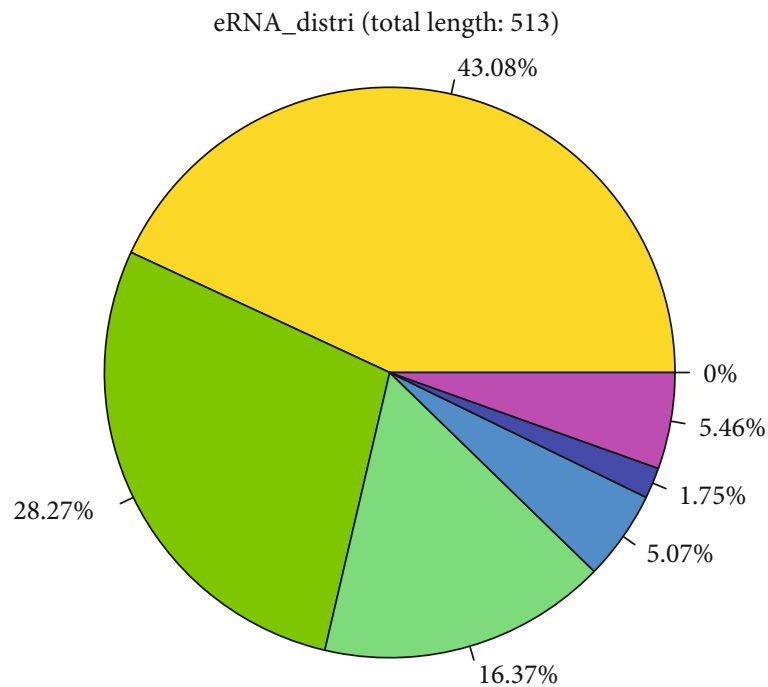

$\begin{array}{ll}\square & 0-500 \\ \square \quad 501-1000 \\ \square \quad 1001-2000 \\ \square \quad 2001-3000\end{array}$

$\square \quad 3001-4000$

ロ 4001-5000

$>5000$

(e)

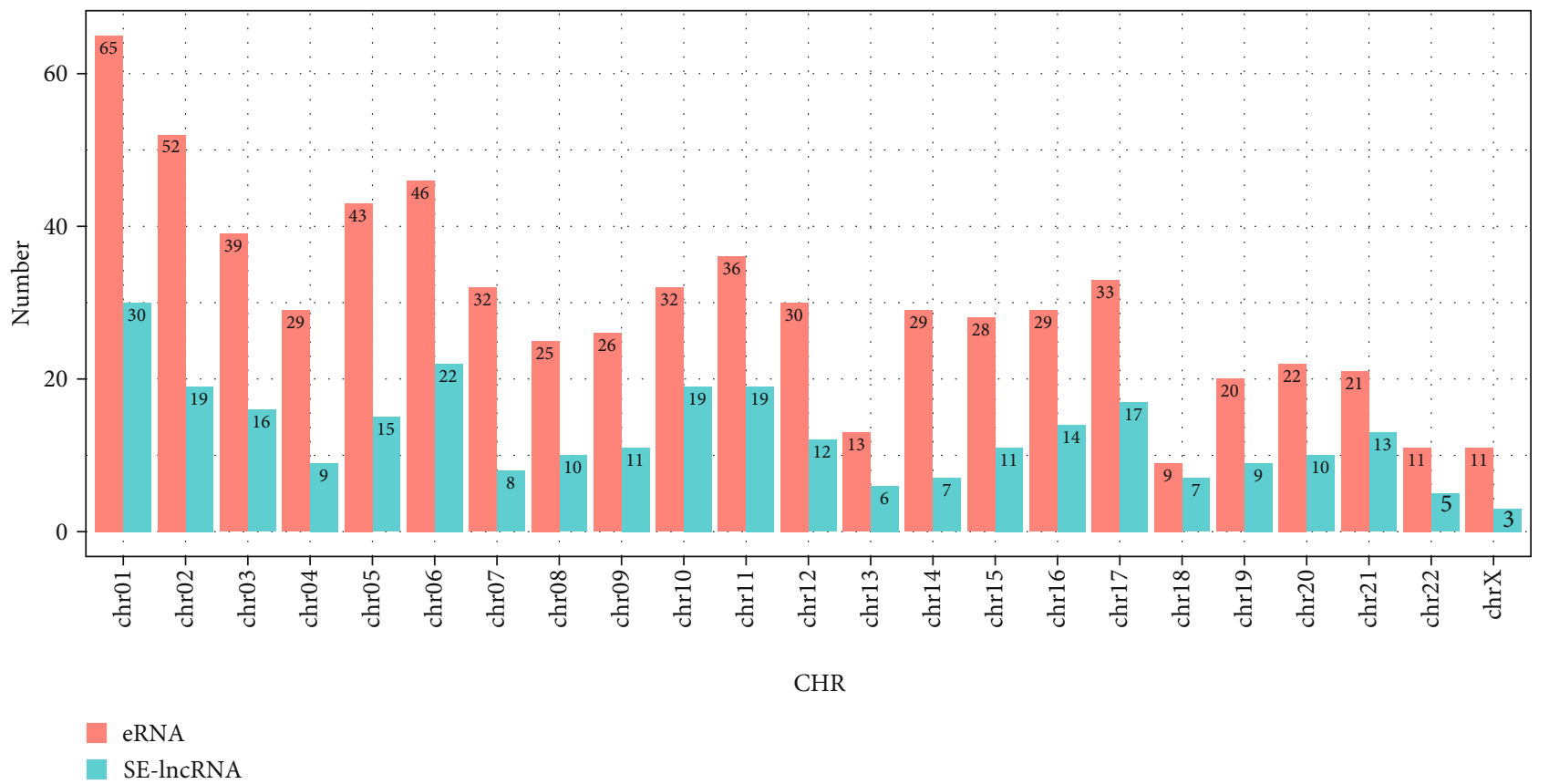

(f)

FIgURE 2: Overview and bioinformatics analysis of eRNAs and SE-lncRNA expression in prostate cancer. (a) Box plot of signals of eRNAs in a microarray that were transcribed from active open chromatin and closed chromatin in LNCaP cells. (b) Barplot of signals of eRNAs and SElncRNAs in a microarray. (c) Circos diagram that shows the chromosome distribution of differentially expressed SE-lncRNAs in a microarray. $(\mathrm{d}-\mathrm{f})$ The distributions of differentially expressed eRNAs and SE-lncRNAs in a microarray were analyzed based on three criteria: classification (d), length (e), and chromosome locus (f).

enhancers were in open chromatin regions, and 82,547 active enhancers were in closed chromatin regions [14]. We also analyzed the expression of eRNAs transcribed from open and closed chromatin regions. Obvi- ously, eRNAs that were transcribed from open chromatin (average signal: 6.25) exhibited increased expression compared with those from closed chromatin (average signal: 5.18 ) in LNCaP cells (Figure 2(a)). 
3.3. Identification of Differentially Expressed SE-lncRNAs in Prostate Cancer. Superenhancers are clusters of enhancers that are formed by binding high levels of master transcription factors and mediator coactivators, which drive high levels of expression of genes that encode key regulators of cell identity [41]. dbSUPER is an integrated and interactive database of superenhancers [42]. To identify SE-lncRNAs contained in the microarray, we downloaded superenhancers from dbSUPER. All the identified superenhancers that overlapped lncRNAs contained in the microarray were further identified as SE-lncRNAs. A total of 14213 SE-lncRNAs were identified in the microarray; 292 differentially expressed SE-lncRNAs were then identified by Student's $t$-test using the threshold of $p$ value $<0.05$ and fold change $\geq 2$ (Figure 2(c)). The distributions of differentially expressed SE-lncRNAs were analyzed, based on three criteria: classification, length, and chromosome locus (Figures 2(d)-2(f)).

To explore whether SE-lncRNAs had higher expression than eRNAs, we also analyzed the expression of all eRNAs and SE-lncRNAs. As expected, the average signal of SElncRNA (average signal: 5.90) was higher than that of eRNA in both tumor and corresponding adjacent normal tissues $(p$ value $\leq 0.0001$ ) (Figure 2(b)), although the differences were not very huge.

\subsection{Potential Target Genes of the Differentially Expressed} eRNAs Were Integrated with Differentially Expressed $m R N A s$. Although the role of most eRNAs in the regulation of gene expression remains unclear, it is considered a hallmark of functionally active enhancers. Other studies suggested that eRNAs mostly in cis to promote enhancerpromoter looping or recruit transcription factors (TFs) to specific enhancers $[24,28,43-45]$. The nearby genes of the differentially expressed eRNAs were defined as potential target genes. If an eRNA expressed differentially with its target gene concordantly, eRNA may play a regulatory role in its target gene expression. So, we integrated the target genes of eRNA with the mRNAs screened by the same microarray assay. A total of 325 differentially expressed target genes were identified for 295 differentially expressed eRNAs. Most of this eRNA $(254,86.1 \%)$ and related target genes (279, $85.8 \%$ ) differentially expressed the same trend, which illustrated that eRNAs were associated with prostate cancer in the regulation of gene expression (Figure 3(a), Table 2, Supplementary Table 4).

3.5. Validation of the Differentially Expressed eRNAs with TCGA Data. To verify the microarray results, we downloaded RNA-seq data $(n=33)$ from TCGA (https://tcgadata.nci.nih.gov/tcga/) generated with nonrecurrent $\mathrm{PCa}$ tumors and normal tissues. Integrated analysis identified that 10144 eRNAs were in both the RNA-seq and the microarray data. A comparison of the differentially expressed genes in the microarray and RNA-seq showed that 267 differentially expressed eRNAs were identified in both the microarray and RNA-seq data (Supplementary Figure 1, Supplementary Table 2). One hundred and eleven eRNAs and their target genes were differentially expressed together, 95 of the 111 eRNAs had the same differentially expressed trend with the target gene, and the other 16 eRNAs were expressed at a low level in RNA-seq data.

3.6. Validation of the Microarray Data with $q R T-P C R$. To further verify the accuracy and reliability of the eRNA microarray results, six eRNAs and partial target genes and two PCa-associated genes (MYC and AR) were selected for a qRT-PCR assay. The housekeeping gene $\beta$-actin was used to normalize the expression level. The qRT-PCR results revealed that the 11 transcript level expressions were remarkably consistent with the microarray data (Figure 3(b)). This confirmed that the microarray data and the analysis based on these data were reliable and accurate, especially the prediction of the target genes of eRNAs. LAMB3, TNFAIP3, and HIST1H2BB were the target genes of ENST00000603283, ENST00000431144, and NONHSAT252076, respectively. LAMB3 was a gene known to be associated with prostate cancer in GeneCards, which is a well-known database that contains gene functions (https:// www.genecards.org/).

3.7. Functional Enrichment Analysis of the Target Genes of eRNAs. To explore the functional implication of the target genes of eRNAs, we performed a functional enrichment analysis of the KEGG pathway using DAVID (https://david .ncifcrf.gov/) and GO by clusterProfiler, which are in the R package. A total of 20 KEGG pathways related to biological pathways were significantly enriched, and the results of the analysis revealed significant pathways were mainly related to prostate cancer (Table 3, Figure 3(d)). A total of $702 \mathrm{GO}$ were significantly enriched, which included biological processes $(n=614)$, cellular components $(n=46)$, and molecular functions $(n=42)$ (Figure 3(c)). Differential expression of multiple target genes associated with inflammation, cellular adhesion, and apoptotic was observed.

We further analyzed the target genes of eRNAs using the GeneAnalytics tool of GeneCards (https://www.genecards .org/). Seventeen target genes were known to be associated with PCa (Table 4), 35 target genes were house-keeping genes, and 31 target genes were abundant genes in multiple organizations. The 17 genes associated with PCa were mainly involved in cancer and prostate cancer-related pathways, which include the prostate cancer pathway, focal adhesion pathway, PI3K-Akt signaling pathway, regulation of actin cytoskeleton pathway, ECM-receptor interaction pathway, proteoglycans in cancer pathway, pathways in cancer, and AMPK signaling pathway. The 17 genes associated with eRNAs may play a role in a similar way in vivo.

3.8. Analysis and Construction of eRNAs That Were Involved in Transcriptional Regulation Networks. eRNAs are required for transcriptional activation, but their functional involvement is unclear. Previous studies suggested that eRNAs may promote enhancer-promoter looping or recruit transcription factors (TFs) to specific enhancers [31, 32]. Therefore, we investigated whether eRNAs and related enhancers were involved in transcriptional regulation. Motif analysis was done using HOMER (http://homer.ucsd.edu/homer/) on the promoters of target genes and differentially expressed 


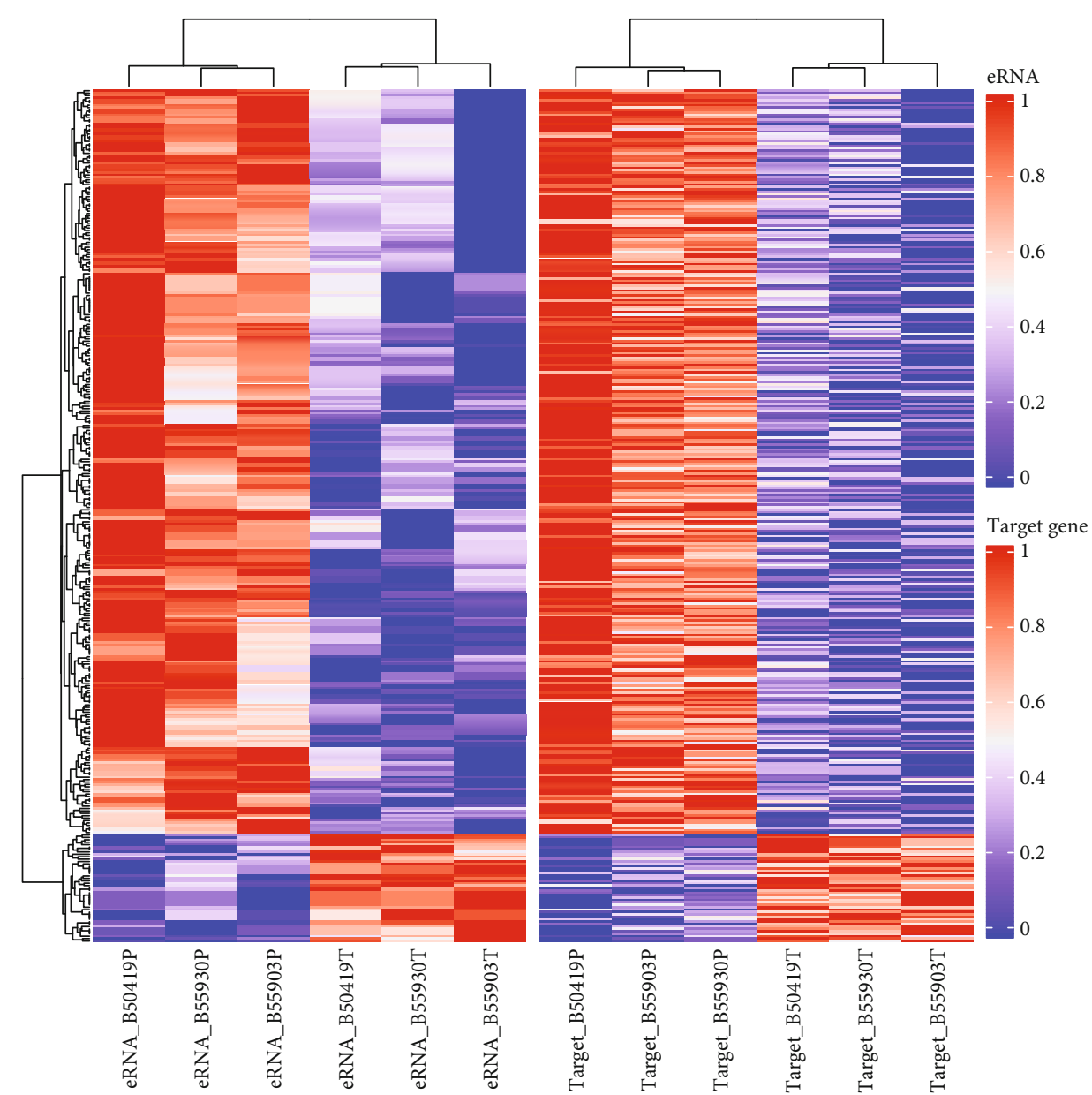

(a)

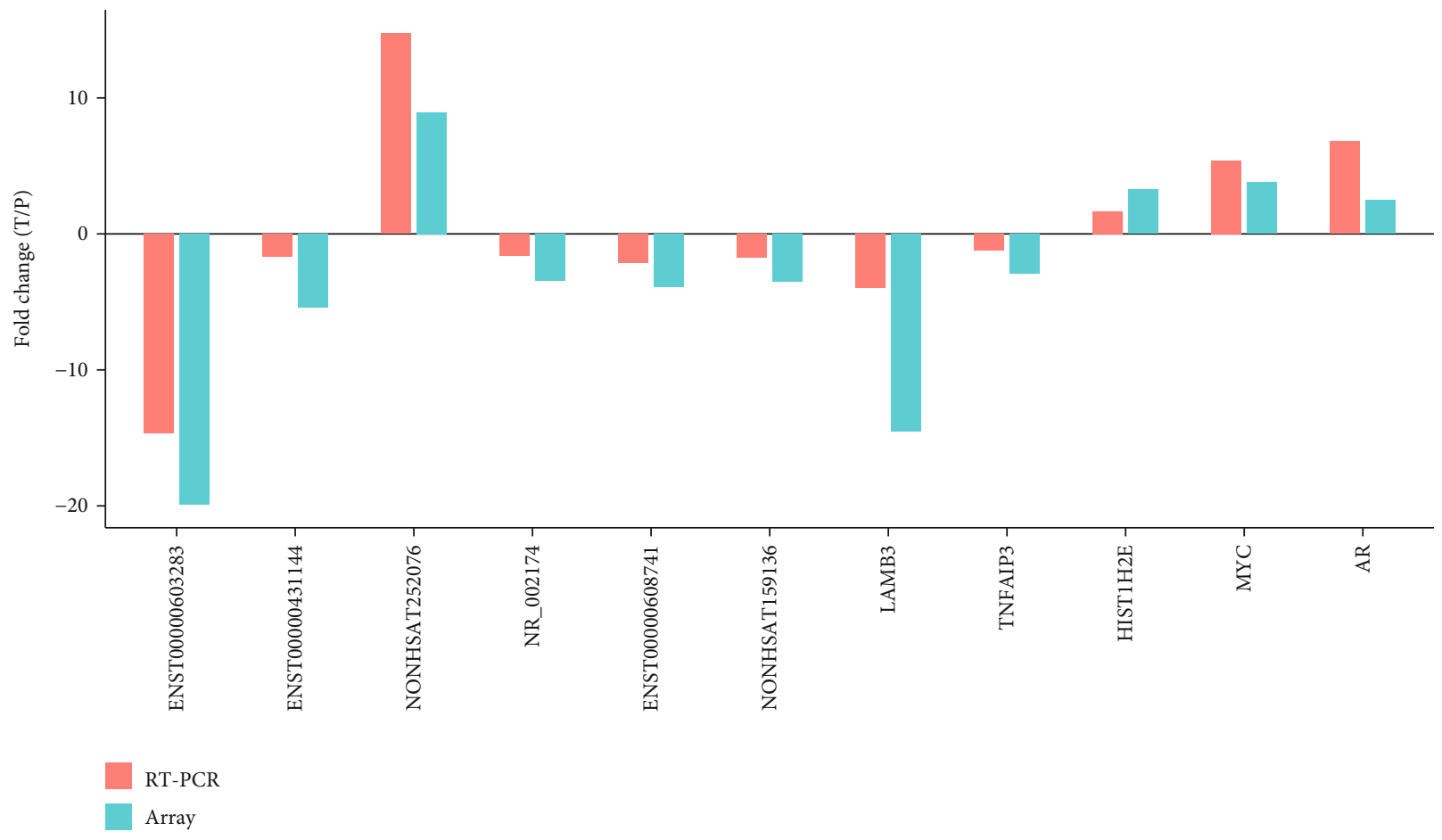

(b)

Figure 3: Continued. 


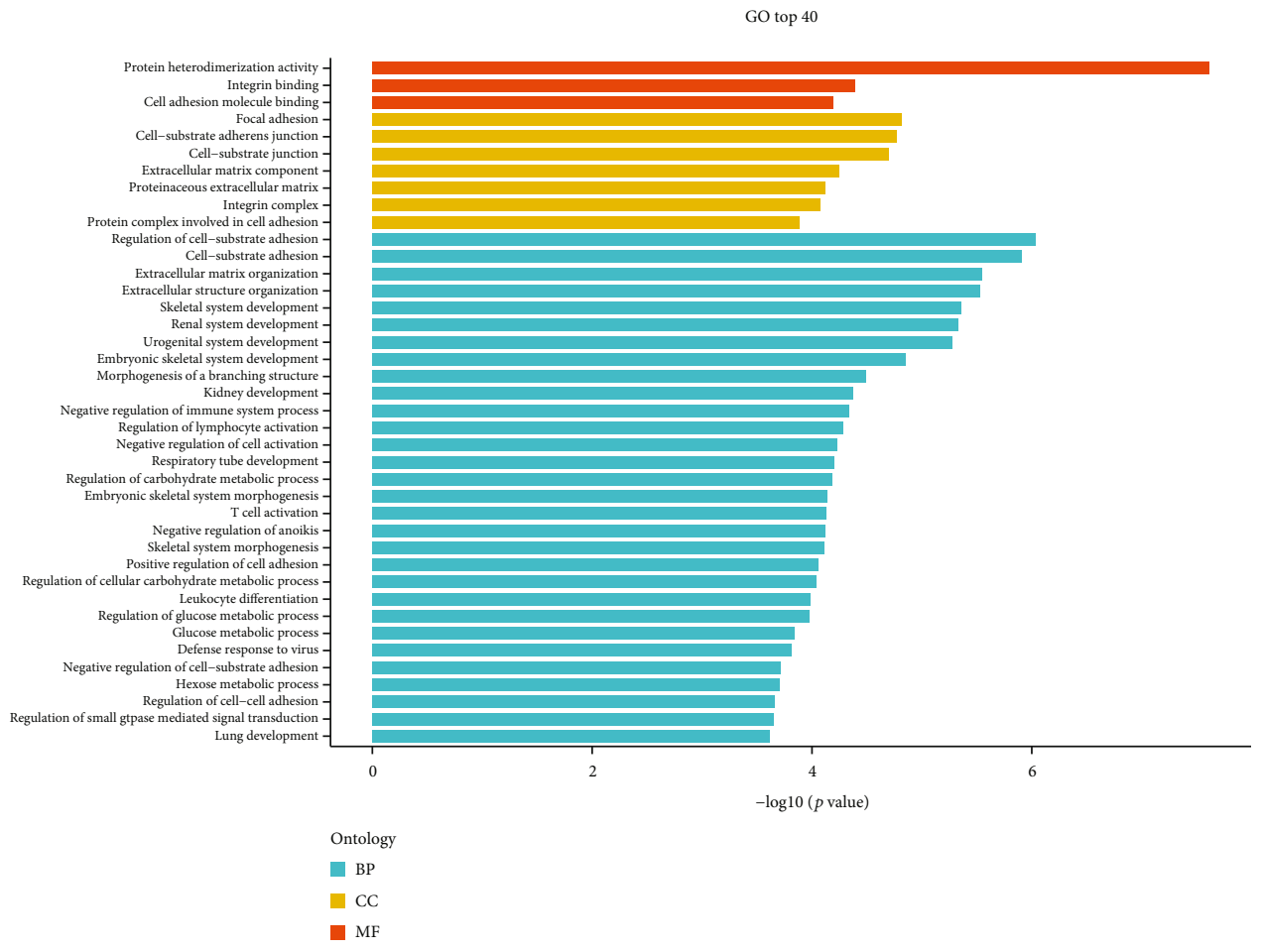

(c)

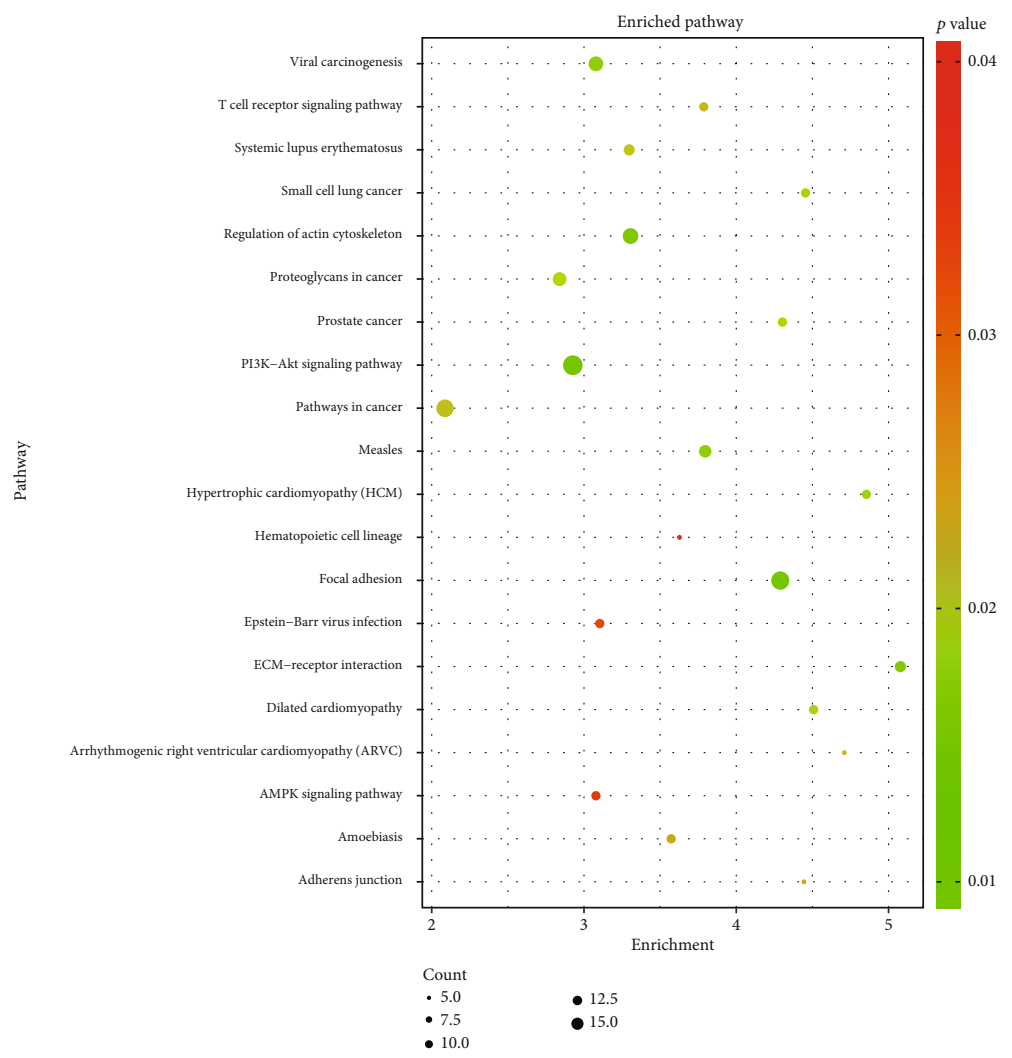

(d)

FIGURE 3: Bioinformatics analysis of target genes of differentially expressed eRNAs in a microarray and validation of selected genes by qRTPCR. (a) Heat map that shows differentially expressed eRNAs and the positive differentially expressed target genes associated with them. (b) The barplot that shows the fold changes of RT-PCR and the microarray assay. The eRNA array data were validated by a RT-PCR assay. (c) Top 40 enriched gene ontology terms of target genes. (d) Top 20 enriched pathways of target genes. The prostate cancer-related pathway was enriched. 
TABLE 2: The top five up and down eRNAs differentially expressed the same trend with the target gene.

\begin{tabular}{lccccccc}
\hline eRNA accession & $p$ value & Fold change & Regulation & Target gene & $p$ value & Fold change & Regulation \\
\hline ENST00000624982 & 0.027 & 21.56 & Down & IL10RA & 0.046 & 2.76 & Down \\
ENST00000458250 & 0.041 & 20.57 & Down & HSD11B1 & 0.004 & 5.14 & Down \\
ENST00000603283 & 0.020 & 19.88 & Down & LAMB3 & 0.024 & 14.53 & 17.09 \\
lnc-SEL1L2-3:1 & 0.013 & 17.27 & Down & FLRT3 & 0.008 & Down \\
ENST00000504829 & 0.024 & 14.75 & Down & SNCAIP & 0.037 & 6.80 & Down \\
NONHSAT252076 & 0.014 & 8.94 & Up & HIST1H1T & 0.037 & 8.79 & Up \\
NONHSAT219894 & 0.008 & 6.12 & Up & CORO2A & 0.025 & 2.11 & Up \\
NONHSAT175755 & 0.030 & 4.68 & Up & SLC25A10 & 0.007 & 2.64 & Up \\
NONHSAT056617 & 0.001 & 4.60 & Up & FASN & 0.022 & 5.44 & Up \\
NONHSAT233757 & 0.025 & 4.40 & Up & PLEKHH1 & 0.046 & 3.37 & Up \\
\hline
\end{tabular}

TABLE 3: Enriched pathway of the target genes of differentially expressed eRNAs in a microarray.

\begin{tabular}{|c|c|c|c|c|}
\hline Term & Pathway & Gene counts & $p$ value & Enrichment \\
\hline hsa04510 & Focal adhesion & 14 & $\leq 0.001$ & 4.29 \\
\hline hsa04151 & PI3K-Akt signaling pathway & 16 & $\leq 0.001$ & 2.93 \\
\hline hsa04810 & Regulation of actin cytoskeleton & 11 & 0.002 & 3.31 \\
\hline hsa04512 & ECM-receptor interaction & 7 & 0.002 & 5.08 \\
\hline hsa05203 & Viral carcinogenesis & 10 & 0.005 & 3.08 \\
\hline hsa05215 & Prostate cancer & 6 & 0.012 & 4.30 \\
\hline hsa05205 & Proteoglycans in cancer & 9 & 0.013 & 2.84 \\
\hline hsa05200 & Pathways in cancer & 13 & 0.020 & 2.09 \\
\hline hsa04520 & Adherens junction & 5 & 0.025 & 4.44 \\
\hline hsa04152 & AMPK signaling pathway & 6 & 0.044 & 3.08 \\
\hline
\end{tabular}

eRNA-related enhancers in a microarray to predict $\mathrm{TF}$ motifs. If the paired eRNA (enhancer) target gene shared the same TF-binding motifs, the eRNA may promote recruited TFs or enhancer-promoter looping. To confirm this, we downloaded the chromatin interaction region data generated with 3C, 4C, and 5C and a ChIA-PET assay from the DENdb database, and we integrated these with previous eRNA- (enhancer-) TF target gene results. With the integrated results, we constructed an eRNA involved in the transcriptional regulation network that was based on an eRNA(enhancer-) TF target gene coordination (Figure 4). eRNA(enhancer-) TF target gene coordinations that were in the top 2 degrees of enhancers are shown in Table 5. There were 34 eRNA- (enhancer-) TF target gene coordinations, which was $23 \%$ of all 147 coordinations. The eRNA-related enhancers with the most degrees were ENST00000448942 (gene name: WAKMAR2-203, from ENSEMBL) and lncPERP-2:25 (gene name: lnc-PERP-2, from LNCipedia), which had the same target gene TNFAIP3. The protein encoded by this gene was a zinc finger protein and ubiquitin-editing enzyme, which has been shown to inhibit NF-kappa B activation and TNF-mediated apoptosis. NR_ 146633 was the second important eRNA in the network, which was a noncoding transcript of SUPT3H; NR_146633 was longer than the coding transcripts. SUPT3H was involved in the C-MYC pathway and transcriptional misregulation in the cancer pathway. Perhaps, NR_146633 was expressed with the coding transcripts concordantly to regulate downstream genes.

3.9. CTCF May Mediate Chromatin Looping. CTCF is a highly conserved gene that displays close to $100 \%$ homology among mice, chickens, and humans [46]. CTCF is a wellknown insulator protein that possesses enhancer blocking functions and barrier functions, but CTCF plays a critical role in mediating chromatin looping rather than functioning as an insulator [50]. It can act both as a transcriptional repressor and an activator. To find out whether CTCF contributed to mediating chromatin looping of enhancer-promoters, the CTCF ChIP-seq data for LNCaP cells were downloaded from ENCODE. We measured whether CTCF was enriched in both enhancers and promoters of 147 eRNA(enhancer-) TF target gene coordinations that we analyzed previously. Not surprisingly, we found that CTCF enriched both enhancers and promoters in 30 eRNA- (enhancer-) TF target gene coordinations. CTCF mediated the chromatin looping of enhancer-promoters. The most important enhancers were K562_Enhancer_301676, K562_Enhancer_ 297273, K562_Enhancer_288447, and K562_Enhancer_ 281124; the most important TFs were KLF5, TFAP2C, USF2, ZNF384, and EGR1.

3.10. eRNAs Regulated Androgen Response Genes and Further Affected Prognosis. Androgens and the androgen receptor 
TABLE 4: Seventeen known prostate cancer-associated target genes.

\begin{tabular}{|c|c|c|c|c|c|}
\hline $\begin{array}{l}\text { Target gene } \\
\text { symbol }\end{array}$ & $\begin{array}{l}\text { Target gene } p \\
\text { value }\end{array}$ & $\begin{array}{l}\text { Target gene fold } \\
\text { change }\end{array}$ & $\begin{array}{l}\text { Target gene } \\
\text { regulation }\end{array}$ & eRNA accession & eRNA type \\
\hline FASN & 0.022 & 5.44 & $\mathrm{Up}$ & NONHSAT056617 & Exonic_sense \\
\hline DCXR & 0.005 & 3.85 & Up & $\begin{array}{l}\text { NONHSAT056617; } \\
\text { NONHSAT238284 }\end{array}$ & Exonic_sense; intergenic \\
\hline PYCR1 & 0.028 & 3.16 & Up & $\begin{array}{l}\text { NONHSAT056617; } \\
\text { NONHSAT175755 }\end{array}$ & Exonic_sense; intergenic \\
\hline HOXB13 & 0.017 & 2.43 & Up & NONHSAT175319; NR_110330 & $\begin{array}{c}\text { Exonic_antisense; exonic_ } \\
\text { antisense }\end{array}$ \\
\hline $\mathrm{LCN} 2$ & 0.036 & 17.45 & Down & NONHSAT134945 & Intronic_sense \\
\hline LAMB3 & 0.024 & 14.53 & Down & $\begin{array}{l}\text { ENST00000458250; } \\
\text { ENST00000603283 }\end{array}$ & Intergenic; intergenic \\
\hline IGF2 & 0.027 & 5.07 & Down & NR_028043; NONHSAT158742 & $\begin{array}{c}\text { Exonic_antisense; exonic } \\
\text { antisense }\end{array}$ \\
\hline RIPOR2 & 0.014 & 4.74 & Down & $\begin{array}{c}\text { NONHSAT208884; NR_002174; } \\
\text { NONHSAT208894 }\end{array}$ & $\begin{array}{c}\text { Exonic_sense; intergenic; } \\
\text { intergenic }\end{array}$ \\
\hline BCL2 & 0.008 & 4.62 & Down & $\begin{array}{l}\text { ENST00000587475; } \\
\text { NONHSAT178558 }\end{array}$ & $\begin{array}{c}\text { Intronic_sense; intronic } \\
\text { sense }\end{array}$ \\
\hline STOM & 0.015 & 4.02 & Down & NR_003191 & Intergenic \\
\hline LY75 & 0.026 & 3.76 & Down & NONHSAT242679 & Intronic_sense \\
\hline ALOX5AP & 0.041 & 3.69 & Down & NR_148425; NONHSAT167432 & Exonic_sense; intergenic \\
\hline FGFR1 & 0.012 & 3.33 & Down & ENST00000519764; NR_148052 & Intergenic; exonic_sense \\
\hline FOXO1 & 0.018 & 3.05 & Down & NONHSAT165576 & Exonic_antisense \\
\hline PIK3R1 & 0.001 & 2.99 & Down & lnc-SLC30A5-6:1 & Exonic_sense \\
\hline COL4A1 & 0.019 & 2.87 & Down & NONHSAT167267 & Exonic_sense \\
\hline MMP14 & 0.014 & 2.53 & Down & NONHSAT234087 & Exonic_antisense \\
\hline
\end{tabular}

(AR) play significant roles in the growth and progression of PCa. Androgens exert their biological and physiological effects by activating AR transcriptional activity [28]. Here, we investigated the expression of Hallmark "androgen response" genes and their potential regulated eRNAs between tumors and corresponding adjacent normal prostate tissues in a microarray. Fourteen eRNAs and the related "androgen response" genes were expressed differentially. For instance, eRNA ENSG00000225339, which has SPDEF (SAM Pointed Domain Containing ETS Transcription Factor) as a target gene, functions as an androgen-independent transactivator of prostate-specific antigen (PSA) promoters. Its related pathway is regulation of androgen receptor activity. Low expression of UBE2J1 is correlated with poor survival of PCa patients in TCGA data. Overall, these "androgen response" genes and their potential regulated eRNAs may be suitable targets for prognostic biomarkers or for developing therapies.

\section{Discussion}

Here, we screened the expression of eRNAs and SElncRNAs in three paired prostate tumor and corresponding adjacent normal prostate tissue samples using a microarray, and we identified 681 prostate cancer-associated differentially expressed eRNAs and 292 SE-lncRNAs. The nearby genes that were differentially expressed with related
eRNAs concordantly were defined as target genes of eRNA. A total of 325 target genes for 295 differentially expressed eRNAs were identified. The target genes mostly participated in cancer occurrence and developmentrelated pathways, which included prostate cancer, focal adhesion, PI3K-Akt signaling pathway, ECM-receptor interaction, viral carcinogenesis, proteoglycans in cancer, pathways in cancer, adherens junction, and AMPK signaling pathway. GO enrichment analysis revealed target genes that were mainly associated with inflammation, cellular adhesion, and apoptotic. The results of GeneAnalytics analysis produced 17 target genes that were known to be associated with PCa. In our microarray data, AR, MYC, and lncRNA PCA3 were upregulated, and PTEN was downregulated. This and qRT-PCR results confirmed that the microarray data and prediction of target genes were reliable and accurate. ENST00000603283, ENST00000431144, and NONHSAT252076 and their target genes LAMB3, TNFAIP3, and HIST1H2BB, respectively, and the known PCa-associated genes AR and MYC were verified by RT-PCR.

Furthermore, we described an eRNA involved in the transcriptional regulation network based on eRNA(enhancer-) TF target gene coordination, and we found 147 eRNA- (enhancer-) TF target gene coordinations. In the coordination, enhancers and promoters of target genes shared the same TF-binding motifs, and the chromatin interaction of enhancers and promoters was proved by 


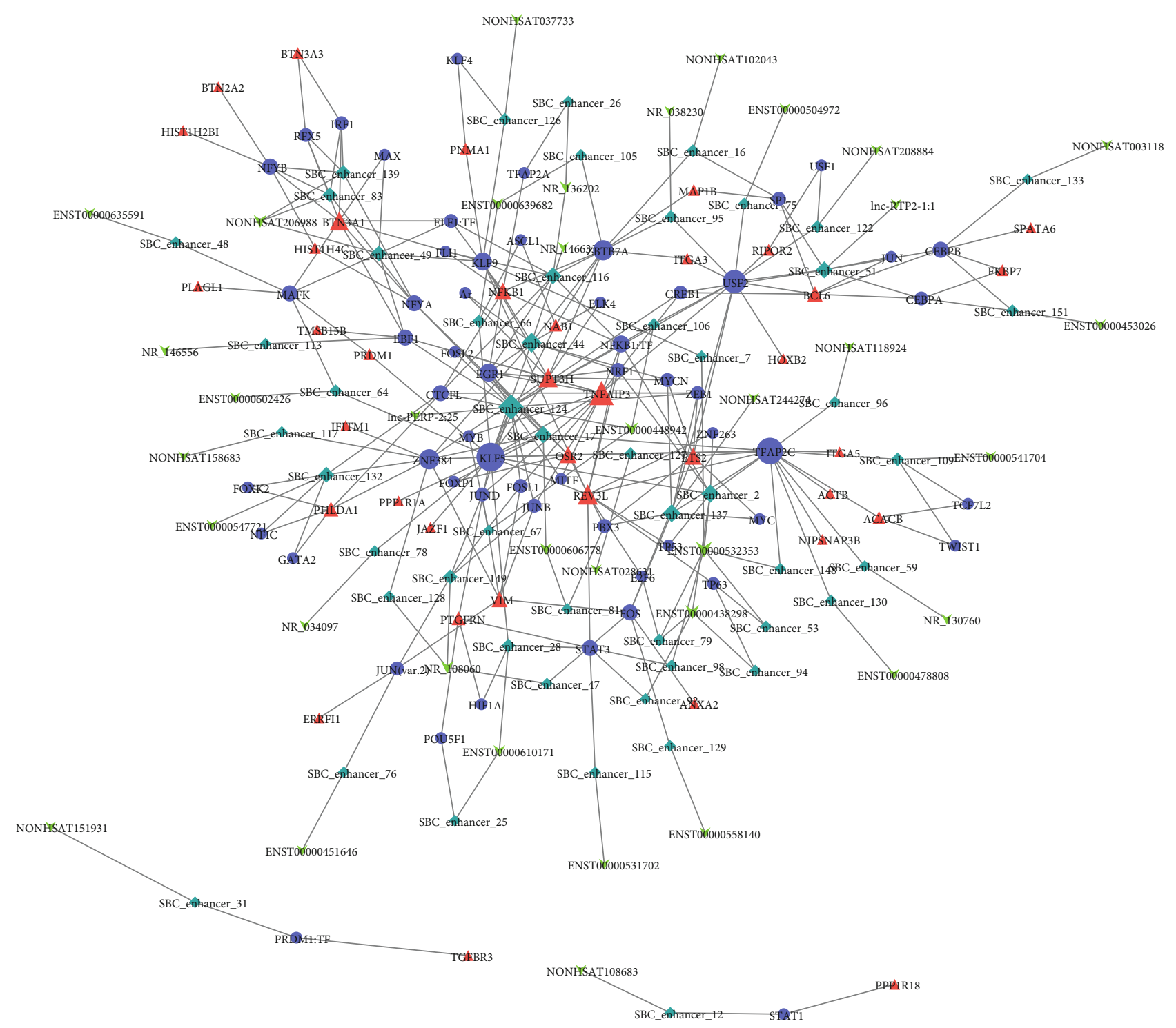

FIgURE 4: Construction of eRNA- (enhancer-) TF target gene coordination network. The network was generated using Cytoscape. The eRNAs (enhancers), TFs (transcription factors), and target genes are marked with squares (green), circles (purple), and triangles (red), respectively. The different sizes of nodes represent different degrees.

3C, 4C, and 5C and a ChIA-PET assay. Also, the CTCF ChIP-seq data revealed that CTCF may be involved in eRNA- (enhancer-) TF target gene regulation networks by mediating chromatin looping; we found 30 potential CTCF-mediated eRNA- (enhancer-) TF target gene coordinations. eRNAs in the coordination promoted the enhancer-promoter looping or recruit transcription factors (TFs) to specific enhancers. We analyzed and constructed the eRNA involved transcriptional regulatory network using gene expression profiling data, transcription factor binding data, and chromosome interaction data.

Prostate cancer is initially androgen-dependent, but the effectiveness of androgen receptor (AR) inhibitors in recurrent disease is variable. The study of androgendependent prostate cancer transcriptional regulatory networks may help to elucidate the molecular mechanism of incipient prostate cancer and provide a foundation for the optimization of prostate cancer therapy strategies and development of drugs. The active eRNAs identified in LNCaP cells were positively correlated with our data of active eRNAs identified in PC-3 cells, mainly because the patients sampled for the microarray assay were androgen-dependent. LNCaP cells are androgen-sensitive human prostate adenocarcinoma cells, but PC-3 cells are derived from bone metastatic sites of prostate cancer patients that are recognized as androgen-independent. This result revealed that the function of eRNA and the transcriptional regulatory mechanism were different between androgen-dependent and androgen-independent prostate cancers. Our study was limited by relatively small sample size; so to validate the microarray results, we compared our data with the TCGA RNA-seq data generated with nonrecurrent $\mathrm{PCa}$ patients, and we verified 11 transcripts using qRT-PCR. We also found that eRNAs 
TABLE 5: The top 2 degrees of enhancer in the network.

\begin{tabular}{|c|c|c|c|}
\hline Enhancer accession & $\mathrm{TF}$ & eRNA accession & Target gene \\
\hline K562_enhancer_301676 & FOSL2 & ENST00000448942 & TNFAIP3 \\
\hline K562_enhancer_301676 & USF2 & ENST00000448942 & TNFAIP3 \\
\hline K562_enhancer_301676 & KLF5 & ENST00000448942 & TNFAIP3 \\
\hline K562_enhancer_301676 & ZEB1 & ENST00000448942 & TNFAIP3 \\
\hline K562_enhancer_301676 & EGR1 & ENST00000448942 & TNFAIP3 \\
\hline K562_enhancer_301676 & JUND & ENST00000448942 & TNFAIP3 \\
\hline K562_enhancer_301676 & JUNB & ENST00000448942 & TNFAIP3 \\
\hline K562_enhancer_301676 & MITF & ENST00000448942 & TNFAIP3 \\
\hline K562_enhancer_301676 & CTCFL & ENST00000448942 & TNFAIP3 \\
\hline K562_enhancer_301676 & FOSL1 & ENST00000448942 & TNFAIP3 \\
\hline K562_enhancer_301676 & EBF1 & ENST00000448942 & TNFAIP3 \\
\hline K562_enhancer_301676 & CREB1 & ENST00000448942 & TNFAIP3 \\
\hline K562_enhancer_301676 & MITF & lnc-PERP-2:25 & TNFAIP3 \\
\hline K562_enhancer_301676 & JUND & lnc-PERP-2:25 & TNFAIP3 \\
\hline K562_enhancer_301676 & NFKB1 & lnc-PERP-2:25 & TNFAIP3 \\
\hline K562_enhancer_301676 & KLF5 & lnc-PERP-2:25 & TNFAIP3 \\
\hline K562_enhancer_301676 & ZEB1 & lnc-PERP-2:25 & TNFAIP3 \\
\hline K562_enhancer_301676 & FOSL2 & lnc-PERP-2:25 & TNFAIP3 \\
\hline K562_enhancer_301676 & EGR1 & lnc-PERP-2:25 & TNFAIP3 \\
\hline K562_enhancer_301676 & USF2 & lnc-PERP-2:25 & TNFAIP3 \\
\hline K562_enhancer_301676 & FOSL1 & lnc-PERP-2:25 & TNFAIP3 \\
\hline K562_enhancer_301676 & CTCFL & lnc-PERP-2:25 & TNFAIP3 \\
\hline K562_enhancer_301676 & EBF1 & lnc-PERP-2:25 & TNFAIP3 \\
\hline K562_enhancer_301676 & CREB1 & lnc-PERP-2:25 & TNFAIP3 \\
\hline K562_enhancer_301676 & JUNB & lnc-PERP-2:25 & TNFAIP3 \\
\hline K562_enhancer_288447 & NRF1 & NR_146633 & SUPT3H \\
\hline K562_enhancer_288447 & ELK4 & NR_146633 & SUPT3H \\
\hline K562_enhancer_288447 & $\mathrm{AR}$ & NR_146633 & SUPT3H \\
\hline K562_enhancer_288447 & EGR1 & NR_146633 & SUPT3H \\
\hline K562_enhancer_288447 & ZNF384 & NR_146633 & SUPT3H \\
\hline K562_enhancer_288447 & MYCN & NR_146633 & SUPT3H \\
\hline K562_enhancer_288447 & ZBTB7A & NR_146633 & SUPT3H \\
\hline K562_enhancer_288447 & KLF9 & NR_146633 & SUPT3H \\
\hline K562_enhancer_288447 & KLF5 & NR_146633 & SUPT3H \\
\hline
\end{tabular}

transcribed from open chromatin had increased expression compared with that transcribed from closed chromatin in LNCaP cells, which was consistent with the basic rules of transcription. Although the mechanisms of our eRNAs should be validated further, our results suggested a new perspective and clues for developing medicines and therapies for prostate cancer; for instance, ENSG00000225339 regulated a prognostic marker gene SPDEF positively that may also serve as a prognostic marker to promote more accurate prediction.

\section{Conclusions}

A comprehensive set of expressions of eRNAs and SElncRNAs was analyzed in PCa. The functions of eRNAs related to $\mathrm{PCa}$ were predicted by a series of bioinformatics analyses. These results furthered our understanding of PCa and should help to promote precision medicine.
Abbreviations
PCa: $\quad$ Prostate cancer
lncRNA: Long noncoding RNA
eRNA: $\quad$ Enhancer RNA
SE-lncRNA: Superenhancer lncRNA
SE: $\quad$ Superenhancers
CTCF: CCCTC-binding factor
TF: $\quad$ Transcription factor
ENCODE: Encyclopedia of DNA elements
DHS: DNase I hypersensitivity sites
STARR-seq: Self-transcribing active regulatory region sequencing 


$\begin{array}{ll}\text { p53BER: } & \text { p53-bound enhancer regions } \\ \text { ER: } & \text { Estrogen receptor } \\ \text { AR: } & \text { Androgen receptor } \\ \text { NCBI: } & \text { National Center for Biotechnology } \\ & \text { Information } \\ \text { GO: } & \text { Gene Ontology } \\ \text { KEGG: } & \text { Kyoto Encyclopedia of Genes and Genomes } \\ \text { DAVID: } & \text { Database for Annotation Visualization and } \\ & \text { Integrated Discovery } \\ \text { TCGA: } & \text { The Cancer Genome Atlas } \\ \text { RPKM: } & \text { Reads per kilobase per million mapped reads } \\ \text { PSA: } & \text { Prostate-specific antigen. }\end{array}$

\section{Data Availability}

The data used to support the findings of this study are available in the GEO database (GSE155056) or from the corresponding author upon request.

\section{Conflicts of Interest}

Our study has no conflicts of interest to disclose.

\section{Authors' Contributions}

Xiaona Zhang, Panpan Pang, and Min Jiang are co-first authors.

\section{Supplementary Materials}

Supplementary Figure 1. Comparison of the differentially expressed eRNAs in the microarray and RNA-seq from TCGA. Venn diagram showing the overlap of the differentially expressed eRNAs identified by the microarray generated in our lab and RNA-seq from TCGA. Supplementary Table 1. All the primer sequences used in qRT-PCR assay. Supplementary Table 2 . The fold changes and $p$ values of differentially expressed eRNAs in RNA-seq data downloaded from TCGA and microarray data generated in our lab. List of differentially expressed eRNAs in microarrays and TCGA (from RNA-seq). Supplementary Table 3. The raw data of qRT-PCR. Supplementary Table 4 . The expression of differentially expressed eRNAs and the same trend differentially expressed target genes and the expression of differentially expressed SE-lncRNAs and the same trend differentially expressed target genes in the microarray data. (Supplementary Materials)

\section{References}

[1] F. Kopp and J. T. Mendell, "Functional classification and experimental dissection of long noncoding RNAs," Cell, vol. 172, no. 3, pp. 393-407, 2018.

[2] I. Ulitsky and D. P. Bartel, "lincRNAs: genomics, evolution, and mechanisms," Cell, vol. 154, no. 1, pp. 26-46, 2013.

[3] N. Brockdorff, A. Ashworth, G. F. Kay et al., "Conservation of position and exclusive expression of mouse Xist from the inactive X chromosome," Nature, vol. 351, no. 6324, pp. 329-331, 1991.
[4] N. Dimitrova, J. R. Zamudio, R. M. Jong et al., "LincRNA-p21 activates $\mathrm{p} 21$ in cis to promote polycomb target gene expression and to enforce the G1/S checkpoint," Molecular Cell, vol. 54, no. 5, pp. 777-790, 2014.

[5] M. Eißmann, T. Gutschner, M. Hämmerle et al., "Loss of the abundant nuclear non-coding RNA MALAT1 is compatible with life and development," RNA Biology, vol. 9, no. 8, pp. 1076-1087, 2012.

[6] T. Derrien, R. Johnson, G. Bussotti et al., "The GENCODE v7 catalog of human long noncoding RNAs: analysis of their gene structure, evolution, and expression," Genome Research, vol. 22, no. 9, pp. 1775-1789, 2012.

[7] F. de Santa, I. Barozzi, F. Mietton et al., "A large fraction of extragenic RNA pol II transcription sites overlap enhancers," PLoS Biology, vol. 8, no. 5, article e1000384, 2010.

[8] U. A. Ørom, T. Derrien, M. Beringer et al., "Long noncoding RNAs with enhancer-like function in human cells," Cell, vol. 143, no. 1, pp. 46-58, 2010.

[9] T. K. Kim, M. Hemberg, J. M. Gray et al., "Widespread transcription at neuronal activity-regulated enhancers," Nature, vol. 465, no. 7295, pp. 182-187, 2010.

[10] D. Tuan, S. Kong, and K. Hu, "Transcription of the hypersensitive site HS2 enhancer in erythroid cells," Proceedings of the National Academy of Sciences of the United States of America, vol. 89, no. 23, pp. 11219-11223, 1992.

[11] H. Chen, G. du, X. Song, and L. Li, "Non-coding transcripts from enhancers: new insights into enhancer activity and gene expression regulation," Genomics Proteomics Bioinformatics, vol. 15, no. 3, pp. 201-207, 2017.

[12] J. Banerji, S. Rusconi, and W. Schaffner, "Expression of a $\beta$ globin gene is enhanced by remote SV40 DNA sequences," Cell, vol. 27, no. 2, pp. 299-308, 1981.

[13] M. Bulger and M. Groudine, "Functional and mechanistic diversity of distal transcription enhancers," Cell, vol. 144, no. 3, pp. 327-339, 2011.

[14] Y. Liu, S. Yu, V. K. Dhiman, T. Brunetti, H. Eckart, and K. P. White, "Functional assessment of human enhancer activities using whole-genome STARR-sequencing," Genome Biology, vol. 18 , no. 1 , p. $219,2017$.

[15] F. Muerdter, L. M. Boryn, and C. D. Arnold, "STARR-seq principles and applications," Genomics, vol. 106, no. 3, pp. 145-150, 2015.

[16] J. O. Yáñez-Cuna, E. Z. Kvon, and A. Stark, "Deciphering the transcriptional cis-regulatory code," Trends in Genetics, vol. 29, no. 1, pp. 11-22, 2013.

[17] M. Levine, C. Cattoglio, and R. Tjian, "Looping back to leap forward: transcription enters a new era," Cell, vol. 157, no. 1, pp. 13-25, 2014.

[18] C. Buecker and J. Wysocka, "Enhancers as information integration hubs in development: lessons from genomics," Trends in Genetics, vol. 28, no. 6, pp. 276-284, 2012.

[19] C. T. Ong and V. G. Corces, "Enhancer function: new insights into the regulation of tissue-specific gene expression," Nature Reviews Genetics, vol. 12, no. 4, pp. 283-293, 2011.

[20] S. Djebali, C. A. Davis, A. Merkel et al., "Landscape of transcription in human cells," Nature, vol. 489, no. 7414, pp. 101-108, 2012.

[21] H. Wu, A. S. Nord, J. A. Akiyama et al., "Tissue-specific RNA expression marks distant-acting developmental enhancers," PLoS Genetics, vol. 10, no. 9, article e1004610, 2014. 
[22] R. Andersson, C. Gebhard, I. Miguel-Escalada et al., "An atlas of active enhancers across human cell types and tissues," Nature, vol. 507, no. 7493, pp. 455-461, 2014.

[23] M. T. Y. Lam, H. Cho, H. P. Lesch et al., "Rev-Erbs repress macrophage gene expression by inhibiting enhancer-directed transcription," Nature, vol. 498, no. 7455, pp. 511-515, 2013.

[24] C. A. Melo, J. Drost, P. J. Wijchers et al., "eRNAs are required for p53-dependent enhancer activity and gene transcription," Molecular Cell, vol. 49, no. 3, pp. 524-535, 2013.

[25] W. Li, D. Notani, Q. Ma et al., "Functional roles of enhancer RNAs for oestrogen-dependent transcriptional activation," Nature, vol. 498, no. 7455, pp. 516-520, 2013.

[26] K. Mousavi, H. Zare, S. Dell'Orso et al., “eRNAs promote transcription by establishing chromatin accessibility at defined genomic loci," Molecular Cell, vol. 51, no. 5, pp. 606-617, 2013.

[27] B. Fang, L. J. Everett, J. Jager et al., "Circadian enhancers coordinate multiple phases of rhythmic gene transcription in vivo," Cell, vol. 159, no. 5, pp. 1140-1152, 2014.

[28] C. L. Hsieh, T. Fei, Y. Chen et al., "Enhancer RNAs participate in androgen receptor-driven looping that selectively enhances gene activation," Proceedings of the National Academy of Sciences of the United States of America, vol. 111, no. 20, pp. 7319-7324, 2014.

[29] N. Hah, S. Murakami, A. Nagari, C. G. Danko, and W. L. Kraus, "Enhancer transcripts mark active estrogen receptor binding sites," Genome Research, vol. 23, no. 8, pp. 12101223, 2013.

[30] L. Yang, C. Lin, C. Jin et al., "lncRNA-dependent mechanisms of androgen-receptor-regulated gene activation programs," Nature, vol. 500, no. 7464, pp. 598-602, 2013.

[31] N. Hah, C. Benner, L. W. Chong, R. T. Yu, M. Downes, and R. M. Evans, "Inflammation-sensitive super enhancers form domains of coordinately regulated enhancer RNAs," Proceedings of the National Academy of Sciences of the United States of America, vol. 112, no. 3, pp. E297-E302, 2015.

[32] J. F. Xiang, Q. F. Yin, T. Chen et al., "Human colorectal cancerspecific CCAT1-L lncRNA regulates long-range chromatin interactions at the MYC locus," Cell Research, vol. 24, no. 5, pp. 513-531, 2014.

[33] X. Lin, T. J. Spindler, M. A. de Souza Fonseca et al., "Superenhancer-associated LncRNA UCA1 interacts directly with AMOT to activate YAP target genes in epithelial ovarian cancer," iScience, vol. 17, pp. 242-255, 2019.

[34] L. A. Torre, F. Bray, R. L. Siegel, J. Ferlay, J. Lortet-Tieulent, and A. Jemal, "Global cancer statistics, 2012," CA: A Cancer Journal for Clinicians, vol. 65, no. 2, pp. 87-108, 2015.

[35] R. L. Siegel, K. D. Miller, and A. Jemal, "Cancer statistics, 2015,” CA A Cancer Journal for Clinicians, vol. 65, no. 1, pp. 5-29, 2015.

[36] C. Lv, S. Fu, Q. Dong et al., "PAGE4 promotes prostate cancer cells survive under oxidative stress through modulating MAPK/JNK/ERK pathway," Journal of Experimental \& Clinical Cancer Research, vol. 38, no. 1, p. 24, 2019.

[37] H. Ashoor, D. Kleftogiannis, A. Radovanovic, and V. B. Bajic, "DENdb: database of integrated human enhancers," Database, vol. 2015, article bav085, 2015.

[38] The ENCODE Project Consortium, "An integrated encyclopedia of DNA elements in the human genome," Nature, vol. 489, no. 7414, pp. 57-74, 2012.
[39] A. R. Quinlan and I. M. Hall, "BEDTools: a flexible suite of utilities for comparing genomic features," Bioinformatics, vol. 26, no. 6, pp. 841-842, 2010.

[40] D. Vučićević, O. Corradin, E. Ntini, P. C. Scacheri, and U. A. Ørom, "Long ncRNA expression associates with tissuespecific enhancers," Cell Cycle, vol. 14, no. 2, pp. 253-260, 2015.

[41] D. Hnisz, B. J. Abraham, T. I. Lee et al., "super-enhancers in the Control of cell identity and disease," Cell, vol. 155, no. 4, pp. 934-947, 2013.

[42] A. Khan and X. Zhang, "dbSUPER: a database of superenhancers in mouse and human genome," Nucleic Acids Research, vol. 44, no. D1, pp. D164-D171, 2016.

[43] G. Natoli and J.-C. Andrau, "Noncoding transcription at enhancers: general principles and functional models," Annual Review of Genetics, vol. 46, no. 1, pp. 1-19, 2012.

[44] F. Lai and R. Shiekhattar, "Enhancer RNAs: the new molecules of transcription," Current Opinion in Genetics \& Development, vol. 25, no. 4, pp. 38-42, 2014.

[45] U. A. Ørom and R. Shiekhattar, "Long non-coding RNAs and enhancers," Current Opinion in Genetics \& Development, vol. 21, no. 2, pp. 194-198, 2011.

[46] J. Yang and V. G. Corces, "Chromatin insulators: a role in nuclear organization and gene expression," Advances in Cancer Research, vol. 110, pp. 43-76, 2011. 